\title{
HILLE-WINTNER TYPE COMPARISON THEOREMS FOR SECOND-ORDER ORDINARY DIFFERENTIAL EQUATIONS
}

\author{
G. J. BUTLER ${ }^{1}$
}

\begin{abstract}
Apart from the Sturm theorem, some of the most useful comparison theorems in second-order ordinary linear differential equations are the Taam-Hille-Wintner theorems. In this note we obtain an extension of these theorems to more general nonlinear equations and incidentially settle an open question for the linear case.
\end{abstract}

1. Introduction. The classical Sturm comparison theorem for the secondorder linear scalar ordinary differential equations

$$
\begin{aligned}
\left(R x^{\prime}\right)^{\prime}+p x & =0, \\
\left(r x^{\prime}\right)^{\prime}+q x & =0,
\end{aligned}
$$

has the following well-known consequence concerning the oscillatory character of the equations: If $r, R, p, q$ are continuous functions with $0<r(t) \leqslant$ $R(t), p(t) \leqslant q(t)$ for all $t$ in $\left[t_{0}, \infty\right)$, and if equation (1.1) is oscillatory (all solutions have arbitrarily large zeros on $\left[t_{0}, \infty\right)$ ), then equation (1.2) is also oscillatory.

A neat application of the Sturm theorem gives the so-called Hille-Wintner comparison theorem which is in terms of the integrals of the coefficient functions $p, q$ :

TheOREM 1.1 [5], [11]. Let $r \equiv R \equiv 1$ and let

$$
P(t)=\int_{t}^{\infty} p(s) d s, \quad Q(t)=\int_{t}^{\infty} q(s) d s
$$

exist with $0 \leqslant P(t) \leqslant Q(t)$ for all $t$ in $\left[t_{0}, \infty\right)$. Then if equation (1.1) is oscillatory, so also is equation (1.2).

Taam [10] extended this result to:

THEOREM 1.2. Let $r(t)$ be bounded above on $\left[t_{0}, \infty\right)$. Let $P(t), Q(t)$ exist and let $0<r(t) \leqslant R(t), 0 \leqslant|P(t)| \leqslant Q(t)$ for all $t$ in $\left[t_{0}, \infty\right)$. Then if equation (1.1) is oscillatory, so also is equation (1.2).

It has remained an open question whether or not the boundedness condition in Taam's result can be removed (see [9], [1]).

Received by the editors March 31, 1978 and, in revised form, October 7, 1977 and June 7, 1978.

AMS (MOS) subject classifications (1970). Primary 34C10, 34C15.

Key words and phrases. Oscillation, nonlinear, comparison.

${ }^{1}$ Supported in part by the National Research Council of Canada, Grant No. A-8130. 
Recently, Schrader [8], using boundary-value problem techniques, has shown that Sturm-type comparison theorems hold for very general secondorder nonlinear differential equations, and it is natural to ask for nonlinear extensions of the Taam-Hille-Wintner theorems. In this note we shall obtain such an extension for a certain class of equations. Furthermore, our results, when applied to the linear case, will settle the question referred to above.

2. Statement and proof of result. We consider the equations:

$$
\begin{gathered}
\left(R x^{\prime}\right)^{\prime}+p f(x)=0, \\
\left(r x^{\prime}\right)^{\prime}+q f(x)=0
\end{gathered}
$$

on some interval $\left[t_{0}, \infty\right)$. Such an equation will be called oscillatory if for each $t_{1} \geqslant t_{0}$ all solutions extendable to $\left[t_{1}, \infty\right)$ oscillate (have arbitrarily large zeros on $\left.\left[t_{1}, \infty\right)\right)$.

THEOREM 2.1. Let $r, R, p, q$ be continuous on $\left[t_{0}, \infty\right)$ such that

$$
P(t)=\int_{t}^{\infty} p(s) d s, \quad Q(t)=\int_{t}^{\infty} q(s) d s
$$

exist, and such that $0<r(t) \leqslant R(t),|P(t)| \leqslant Q(t)$ for all $t \in\left[t_{0}, \infty\right)$. Assume that $f$ satisfies the following conditions:

(a) $f$ is continuously differentiable, $x f(x)>0$ for all $x \neq 0, f^{\prime}(x)>0$ for all $x \neq 0$, and either

(b) $f^{\prime}$ is nondecreasing on $[0, \infty)$ and is nonincreasing on $(-\infty, 0]$ or

(c) $\lim _{|x| \rightarrow \infty} f^{\prime}(x)>0$ and $\int_{ \pm 1}^{ \pm \infty} d u / f(u)<\infty$.

Then if equation (2.1) is oscillatory, so also is equation (2.2).

We see that $f(x) \equiv x$ satisfies (a) and (b), and that $f(x) \equiv x^{\alpha}$ satisfies (a), (b) and (c) if $\alpha>1$ is the ratio of odd integers. Hence we have:

COROLlary 2.2. The Taam-Hille-Wintner comparison theorem for (1.1) and (1.2) holds without the restriction that $r$ be bounded, and extends to nonlinear equations of the form (2.1) and (2.2) where $f(x) \equiv x^{\alpha}, \alpha>1$ being the ratio of odd integers.

Conditions (a) and (b) say that $|f|$ is a convex function; conditions (a) and (c) comprise a kind of strong nonlinearity condition on $f$, under which Butler previously obtained a characterization of the oscillatory behavior of the equation

$$
y^{\prime \prime}+a f(y)=0
$$

which we state here as follows:

LEMMA 2.3 [2]. Let $A(t)=\int_{t}^{\infty} a(s) d s$ exist with $A(t) \geqslant 0$ on $\left[t_{0}, \infty\right)$, and let $f$ be strongly nonlinear. Then equation (2.3) is oscillatory if and only if

$$
\int^{\infty}\left[A(s)+\int_{s}^{\infty} A^{2}(u) d u\right] d s=\infty
$$


If we remove the sign condition on $A$, then a necessary condition for (2.3) to be oscillatory is

$$
\int^{\infty}\left[|A(s)|+\int_{s}^{\infty} A^{2}(u) d u\right] d s=\infty
$$

Proof of Theorem 2.1. It will be convenient to separate the proof into three cases:

(i) $\int^{\infty} d u / r(u)=\infty,|f|$ convex (conditions (a) and (b)).

(iii) $\int^{\infty} d u / r(u)=\infty, f$ strongly nonlinear (conditions (a) and (c)).

(iii) $\int^{\infty} d u / r(u)<\infty$.

Case (i). Suppose that equation (2.2) is not oscillatory. Then there is a solution $x(t)$ of (2.2) which is eventually of one sign, and without loss of generality, we may assume that $x(t)>0$ on $\left[t_{0}, \infty\right)$. Put $z(t)=$ $r(t) x^{\prime}(t) / f(x(t))$ to obtain the Riccati equation

$$
z^{\prime}=-q-f^{\prime}(x) z^{2} / r .
$$

Then for $t_{0} \leqslant t \leqslant T$, we have

$$
z(t)=z(T)+\int_{t}^{T} q(s) d s+\int_{t}^{T}\left(f^{\prime}(x(s)) \frac{z^{2}(s)}{r(s)}\right) d s .
$$

Letting $T \rightarrow \infty$ in (2.4), the second term on the right-hand side has the nonnegative limit $Q(t)$ and the third term has either a nonnegative limit or the limit $\infty$. Hence we must have $\lim _{T \rightarrow \infty} z(T)=b$, where $-\infty<b<\infty$.

Next we show that $0 \leqslant b<\infty$. Suppose on the contrary, that $-\infty<b<$ 0 . Then for sufficiently large $t$, say $t \geqslant \bar{t}$, we shall have $r(t) x^{\prime}(t)<0$. If for some $\varepsilon, r(t) x^{\prime}(t) \leqslant-\varepsilon<0$ for $t \geqslant t^{*}$, say, we shall have

$$
x(t)-x\left(t^{*}\right) \leqslant-\varepsilon \int_{t^{*}}^{t} \frac{d s}{r(s)} \rightarrow-\infty \quad \text { as } t \rightarrow \infty,
$$

contradicting $x(t)>0$ on $\left[t_{0}, \infty\right)$. Thus we must have $\varlimsup_{t \rightarrow \infty} r(t) x^{\prime}(t)=0$. We obtain a contradiction as follows (see also [3]): Choose $t_{n}>\bar{t}$ with $t_{n} \uparrow \infty$ so that for sufficiently large $n$ and for $\bar{t}<t<t_{n}$, we have $-1 / n=r\left(t_{n}\right) x^{\prime}\left(t_{n}\right)$ $>r(t) x^{\prime}(t)$. Integrating (2.2) between $t$ and $t_{n}$ where $t<t<t_{n}$, for $n$ large, we obtain

$$
\begin{aligned}
0 & =r\left(t_{n}\right) x^{\prime}\left(t_{n}\right)-r(t) x^{\prime}(t)+\int_{t}^{t_{n}} q(s) f(x(s)) d s \\
& >\int_{t}^{t_{n}} q(s) f(x(s)) d s \\
& =\left(\int_{t}^{t_{n}} q(s) d s\right) f(x(t))+\int_{t}^{t_{n}}\left(\int_{s}^{t_{n}} q(u) d u\right) f(x(s)) \frac{x^{\prime}(s) f^{\prime}(x(s)) d s}{f(x(s))} \\
& =V(t)-\int_{t}^{t_{n}} H(s) V(s) d s,
\end{aligned}
$$


where

$$
V(t)=\left(\int_{t}^{t_{n}} q(s) d s\right) f(x(t)), \quad H(t)=\frac{-x^{\prime}(t) f^{\prime}(x(t))}{f(x(t))}>0 .
$$

From the inequality

$$
V(t)<\int_{t}^{t_{n}} H(s) V(s) d s,
$$

we set

$$
W(t)=\int_{t}^{t_{n}} H(s) V(s) d s
$$

to obtain $W^{\prime}(t)=-H(t) V(t)>-H(t) W(t)$, from which follows

$$
\frac{d}{d t}\left[W(t) \exp \left(\int_{\bar{t}}^{t} H(s) d s\right)\right]>0, \quad \bar{t} \leqslant t<t_{n},
$$

and we deduce that

$$
0=W\left(t_{n}\right) \exp \left(\int_{t}^{t_{n}} H(s) d s\right)>W(t) \exp \left(\int_{\bar{t}}^{t} H(s) d s\right)
$$

which implies that $W(t)$, and hence $V(t)$, is negative on $\left[\bar{t}, t_{n}\right)$. But this in turn implies that $\int_{t}^{t} q(s) d s<0$ for $\bar{t} \leqslant t<t_{n}$, for $n$ sufficiently large, contradicting the nonnegativity of $Q(t)=\int_{t}^{\infty} q(s) d s$.

Hence on taking limits in (2.4) we have

$$
z(t)=b+Q(t)+\int_{t}^{\infty}\left(f^{\prime}(x(s)) z^{2}(s) / r(s)\right) d s,
$$

where $0 \leqslant b<\infty$, as claimed. Now $x^{\prime} / f(x)=z / r$, so that

$$
\int_{c}^{x(t)} \frac{d u}{f(u)}=\int_{t_{0}}^{t}\left(\frac{z(s)}{r(s)}\right) d s
$$

where $c=x\left(t_{0}\right)>0$ (we note from (2.5) that $z(t) \geqslant 0$ and so $x(t) \geqslant x\left(t_{0}\right)$ on $\left.\left[t_{0}, \infty\right)\right)$. Denoting $\int_{c}^{x} d u / f(u)$ by $\Omega(x)$, for $x>0$, and the inverse function of $\Omega$ by $\Gamma$, we have

$$
x(t)=\Gamma\left[\int_{t_{0}}^{t}\left(\frac{z(s)}{r(s)}\right) d s\right]
$$

and we may write $(2.5)$ as

$$
z(t)=b+Q(t)+\int_{t}^{\infty}\left(\frac{z^{2}(s)}{r(s)}\right) f^{\prime}\left(\Gamma\left[\int_{t_{0}}^{s}\left(\frac{z(u)}{r(u)}\right) d u\right]\right) d s
$$

Conditions (a) and (b) imply that $\Gamma$ is monotone increasing from some interval $(-\infty, B)$ to $(0, \infty)(B$ may be $+\infty)$ and that we may write $(2.6)$ as

$$
z=M(z)
$$

where $M$ is a self-map of the set $C$ of continuous functions $u$ with $0<u(t)<$ $z(t), t \in\left[t_{0}, \infty\right) . M$ is monotone in the sense that $M u<M v$ for any $u, v \in C$ with $u<v$, under the natural ordering of $C$. 
Now consider the integral equation

$$
w(t)=b+P(t)+\int_{t}^{\infty}\left(\frac{w^{2}(s)}{R(s)}\right) f^{\prime}\left(\Gamma\left[\int_{t_{0}}^{s}\left(\frac{w(u)}{R(u)}\right) d u\right]\right) d s
$$

which we may write as

$$
w=L(w) .
$$

Since $|P(t)| \leqslant Q(t), 0<r(t) \leqslant R(t)$ on $\left[t_{0}, \infty\right)$, we see that $L$ is a self-map of $D$, the set of continuous functions $u$ with $P(t) \leqslant u(t) \leqslant z(t), t \in\left[t_{0}, \infty\right)$. D is a closed convex subset of $C=C\left[t_{0}, \infty\right)$, the Fréchet space of continuous real-valued functions on $\left[t_{0}, \infty\right)$, with the compact-open topology. Let $w \in D$. Then

$$
|w(t)| \leqslant z(t), \quad|(L w)(t)| \leqslant z(t), \quad t \in\left[t_{0}, \infty\right)
$$

and for $t_{0} \leqslant s<t \leqslant T$, we have

$$
|(L w)(t)-(L w)(s)| \leqslant\left|\int_{s}^{t} p(u) d u\right|+\left|\int_{s}^{t} q(u) d u\right|+|z(t)-z(s)| .
$$

From (2.10) and (2.11), it follows that the functions in the image $L(D)$ of $D$ are uniformly bounded and equicontinuous on compact subintervals of $\left[t_{0}, \infty\right)$. We shall show that $L$ is continuous on $D$. For $w \in \mathcal{C}$, define $\phi(w ; s)$ by

$$
\phi(w ; s)=\left(\frac{w^{2}(s)}{R(s)}\right) f^{\prime}\left(\Gamma\left[\int_{t_{0}}^{s}\left(\frac{w(u)}{R(u)}\right) d u\right]\right)
$$

so that

$$
(L w)(t)=b+P(t)+\int_{t}^{\infty} \phi(w ; s) d s .
$$

Let $w, w_{n} \in \mathcal{C}$ such that $w_{n} \rightarrow w$ uniformly on compact subintervals of $\left[t_{0}, \infty\right)$. Let $I=\left[t_{0}, t_{1}\right], \varepsilon>0$ be given and choose $T_{1}>t_{1}$ so that

$$
\int_{T_{1}}^{\infty}\left(\frac{z^{2}(s)}{r(s)}\right) f^{\prime}\left(\Gamma\left[\int_{t_{0}}^{s}\left(\frac{z(u)}{r(u)}\right) d u\right]\right)<\frac{\varepsilon}{3} .
$$

Noting that $\phi\left(w_{n} ; s\right) \rightarrow \phi(w ; s)$, as $n \rightarrow \infty$, uniformly on $\left[t_{0}, T_{1}\right]$, we may use (2.10), (2.12) (2.13) and the monotonicity of $f^{\prime} \circ \Gamma$ to obtain, for $t \in I$,

$$
\begin{aligned}
\left|(L w)(t)-\left(L w_{n}\right)(t)\right| \leqslant & \left|\int_{t}^{T_{1}}\left(\phi(w ; s)-\phi\left(w_{n} ; s\right)\right) d s\right| \\
& +\int_{T_{1}}^{\infty}\left(|\phi(w ; s)|+\left|\phi\left(w_{n} ; s\right)\right|\right) d s \\
& <\varepsilon / 3+2 \varepsilon / 3<\varepsilon,
\end{aligned}
$$

for $n$ sufficiently large. Thus $L w_{n} \rightarrow L w$, as $n \rightarrow \infty$, uniformly on compact subintervals of $\left[t_{0}, \infty\right)$. It follows that $L$ is continuous on $D$ and so we may apply a corollary of Tychonov's theorem [4, p. 405] to deduce the existence of 
a fixed point $W$ of (2.9). Then

$$
X(t)=\Gamma\left[\int_{t_{0}}^{t}\left(\frac{W(s)}{R(s)}\right) d s\right]
$$

is a nonoscillatory solution of $(2.1)$ on $\left[t_{0}, \infty\right)$. This establishes the theorem for case (i).

Case (ii). We make a standard change of variables

$$
s=\int_{t_{0}}^{t} \frac{d \tau}{r(\tau)}, \quad y(s)=x(t(s))
$$

to transform (2.2) into

$$
\frac{d^{2} y}{d s^{2}}+r(t(s)) q(t(s)) f(y)=0 .
$$

(See, for example, [12, p. 227].)

The oscillation properties of the equation are invariant under this transformation. Putting $a(s)=r(t(s)) q(t(s))$, and making the substitution $u=t(\tau)$, we have

$$
\begin{gathered}
A(s)=\int_{s}^{\infty} a(\tau) d \tau=\int_{s}^{\infty} r(t(\tau)) q(t(\tau)) d \tau \\
=\int_{t(s)}^{\infty} q(u) d u=Q(t(s)) \geqslant 0 \\
\int_{T}^{\infty} A(s) d s=\int_{T}^{\infty} Q(t(s)) d s=\int_{t(T)}^{\infty}\left(\frac{Q(u)}{r(u)}\right) d u \\
\int_{T}^{\infty}\left(\int_{s}^{\infty} A^{2}(\tau) d \tau\right) d s=\int_{t(T)}^{\infty}\left(\frac{1}{r(u)}\right)\left(\int_{u}^{\infty}\left(\frac{Q^{2}(v)}{r(v)}\right) d v\right) d u
\end{gathered}
$$

It follows from Lemma 2.3 and equations (2.14)-(2.17) that equation (2.2) is oscillatory if and only if

$$
\int^{\infty}\left(\frac{1}{r(u)}\right)\left\{Q(u)+\int_{u}^{\infty}\left(\frac{Q^{2}(v)}{r(v)}\right) d v\right\} d u=\infty .
$$

Similarly, we see that a necessary condition for equation (2.1) to be oscillatory is that

$$
\int^{\infty}\left(\frac{1}{R(u)}\right)\left\{|P(u)|+\int_{u}^{\infty}\left(\frac{P^{2}(v)}{R(v)}\right) d v\right\} d u=\infty
$$

Since $0<r \leqslant R, 0 \leqslant|P| \leqslant Q$, we see from (2.18) and (2.19) that the theorem is valid for Case (ii).

Case (iii). We shall show that in this case, condition (a) and the existence of the integral $P(t)$ already imply that the equation (2.1) has a nonoscillatory solution. This is already known in the linear case [7]. Thus the comparison theorem is redundant for this situation. Here the appropriate transformation 
is

$$
s=g(t)=\left(\int_{t}^{\infty} \frac{d \tau}{R(\tau)}\right)^{-1}, \quad y(s)=s x(t) .
$$

If we denote $g\left(t_{0}\right)$ by $s_{0}$ and the inverse function of $g$ by $h$, we find that the transformation changes $(2.1)$ into

$$
\frac{d^{2} y}{d s^{2}}+a(s) f(y / s)=0, \quad s \in\left[s_{0}, \infty\right),
$$

where $a(s)=R(h(s)) p(h(s)) / s^{3}($ see $[12])$.

Choose a constant $k \geqslant 1$ such that

$$
0 \leqslant f^{\prime}(x) \leqslant k \text { whenever } 0 \leqslant x \leqslant 2
$$

and choose $T_{0} \geqslant \max \left(1, s_{0}\right)$ so that

$$
0 \leqslant \hat{P}=\max _{s>T_{0}}|P(h(s))| \leqslant \frac{1}{24 k}
$$

(note $P(t) \rightarrow 0$ as $t \rightarrow \infty$ ).

We shall consider the integral equation

$$
y(T)=1-\int_{T}^{\infty}\left(\int_{s}^{\infty} a(\tau) f\left(\frac{y(\tau)}{\tau}\right) d \tau\right) d s,
$$

which we write as $y=F(y)$. First we make a preliminary estimate.

Let

$$
\begin{aligned}
A(s) & =\int_{s}^{\infty} a(\tau) d \tau=\int_{s}^{\infty}\left(\frac{R(h(\tau)) p(h(\tau))}{\tau^{3}}\right) d \tau \\
& =\int_{h(s)}^{\infty} p(u)\left(\int_{u}^{\infty} \frac{d v}{R(v)}\right) d u \\
& =P(h(s)) \cdot\left(\int_{h(s)}^{\infty} \frac{d v}{R(v)}\right)-\int_{h(s)}^{\infty}\left(\frac{P(u)}{R(u)}\right) d u .
\end{aligned}
$$

Thus $A(s)$ is well defined and satisfies

$$
|A(s)| \leqslant 2 \hat{P} \cdot \int_{h(s)}^{\infty} \frac{d u}{R(u)}=\frac{2 \hat{P}}{s}, \quad \text { for } s \geqslant T_{0} .
$$

Next we denote $\left[T_{0}, \infty\right)$ by $I$ and we let $X$ be the Banach space of bounded continuously differentiable functions $y$ on $I$ such that $t y^{\prime}$ is bounded on $I$. $X$ is normed by $\|y\|=\|y\|_{\infty}+\left\|t y^{\prime}\right\|_{\infty}$, where $\|\cdot\|_{\infty}$ denotes the supremum on I.

Let $B$ be the closed ball of $X$ with center the constant function 1 and radius $\frac{1}{2}$. We shall show that $F$ maps $B$ continuously into a compact subset of itself, which permits us to use the Schauder fixed-point theorem to deduce the existence of $y^{*} \in B$ such that $F\left(y^{*}\right)=y^{*} \cdot y^{*}(s)$ will then be a nonoscillatory solution of (2.20) with a corresponding nonoscillatory solution of (2.1) as claimed. Throughout the following calculations, $t>T_{0} \geqslant 1$. If $y \in B$, we have $|y(t)-1| \leqslant \frac{1}{2}$ and $\left|t y^{\prime}(t)\right| \leqslant \frac{1}{2}$, and so 


$$
\left|\left(\frac{y(t)}{t}\right)^{\prime}\right|=\left|\frac{y^{\prime}(t)}{t}-\frac{y(t)}{t^{2}}\right|<\frac{2}{t^{2}} .
$$

Noting that $0 \leqslant y(t) / t<2 / t \leqslant 2$, and using (2.21) to (2.24), we have

$$
\begin{aligned}
\left|[F(y)]^{\prime}(t)\right| & =\left|\int_{t}^{\infty} a(s) f\left(\frac{y(s)}{s}\right) d s\right| \\
& =\left|A(t) f\left(\frac{y(t)}{t}\right)+\int_{t}^{\infty} A(s) f^{\prime}\left(\frac{y(s)}{s}\right)\left(\frac{y(s)}{s}\right)^{\prime} d s\right| \\
& \leqslant \frac{2 k|A(t)|}{t}+\int_{t}^{\infty}\left(\frac{2 k|A(s)|}{s^{2}}\right) d s<\frac{6 k \hat{P}}{t^{2}}<\frac{1}{4 t}
\end{aligned}
$$

and so

$$
|[F(y)](t)-1| \leqslant 6 k \hat{P} / t<1 / 4 .
$$

From (2.26) and (2.27) we find that $F(y) \in B$ and so $F$ maps $B$ into itself.

Now let $y, z \in B$ with $\|y-z\| \leqslant \varepsilon$. We have

$$
\begin{gathered}
\left|[F(y)]^{\prime}(t)-[F(z)]^{\prime}(t)\right|=\left|\int_{t}^{\infty} a(s)\left\{f\left(\frac{y(s)}{s}\right)-f\left(\frac{z(s)}{s}\right)\right\} d s\right| \\
\leqslant|A(t)|\left|f\left(\frac{y(t)}{t}\right)-f\left(\frac{z(t)}{t}\right)\right|+\int_{t}^{\infty}|A(s)||U(s)| d s,
\end{gathered}
$$

where

$$
U(s)=f^{\prime}\left(\frac{y(s)}{s}\right)\left(\frac{y(s)}{s}\right)^{\prime}-f^{\prime}\left(\frac{z(s)}{s}\right)\left(\frac{z(s)}{s}\right)^{\prime} .
$$

Using (2.21) and the mean value theorem, we have

$$
\left|f\left(\frac{y(t)}{t}\right)-f\left(\frac{z(t)}{t}\right)\right| \leqslant \frac{k|y(t)-z(t)|}{t} \leqslant \frac{k \varepsilon}{t} .
$$

Writing $U(s)$ as

$$
\begin{aligned}
\left\{f^{\prime}\left(\frac{y(s)}{s}\right)\right. & \left.-f^{\prime}\left(\frac{z(s)}{s}\right)\right\}\left(\frac{y(s)}{s}\right)^{\prime} \\
& +f^{\prime}\left(\frac{z(s)}{s}\right)\left\{\frac{y^{\prime}(s)-z^{\prime}(s)}{s}+\frac{z(s)-y(s)}{s^{2}}\right\},
\end{aligned}
$$

we may use (2.21) and (2.25) to obtain

$$
|U(s)| \leqslant\left(\frac{2}{s^{2}}\right) \omega\left(\frac{\varepsilon}{s}\right)+\frac{2 k \varepsilon}{s^{2}} \leqslant\left(\frac{2}{s^{2}}\right)(\omega(\varepsilon)+k \varepsilon),
$$

where $\omega$ is the modulus of continuity of $f^{\prime}$ on [0, 2]. Applying (2.24), (2.29) and (2.30) in (2.28), we find that

$$
\begin{aligned}
\left|[F(y)]^{\prime}(t)-[F(z)]^{\prime}(t)\right| & \leqslant 2 k \hat{P} \varepsilon / t^{2}+4 \hat{P}(\omega(\varepsilon)+k \varepsilon) \int_{t}^{\infty}\left(1 / s^{3}\right) d s \\
& \leqslant 2 \hat{P}(2 k \varepsilon+\omega(\varepsilon)) / t^{2}
\end{aligned}
$$


and, therefore,

$$
|[F(y)](t)-[F(z)](t)| \leqslant 2 \hat{P}(2 k \varepsilon+\omega(\varepsilon)) / t .
$$

It follows from (2.31) and (2.32) that $\|F(y)-F(z)\| \leqslant 4 \hat{P}(2 k \varepsilon+\omega(\varepsilon))$, and so $F$ is a continuous mapping of $B$ into itself.

Finally, let $y \in B$ and let $T_{0} \leqslant s \leqslant T$. Then

$$
\left|[F(y)]^{\prime}(t)-[F(y)]^{\prime}(s)\right|=\left|\int_{s}^{t} a(\tau) f(y(\tau) / \tau) d \tau\right| \leqslant 2 k \int_{s}^{t}|a(\tau)| d \tau .
$$

From (2.33), it follows that the image $F(B)$ of $B$ under $F$ consists of uniformly bounded functions on $I$, with uniformly bounded derivatives on $I$ that are equicontinuous on compact subsets of $I$. Consequently, we may apply the Ascoli theorem to deduce that $F(B)$ has compact closure in $X$. Thus a standard corollary of the Schauder fixed-point theorem may be applied [4, p. 405]. This finishes the treatment of Case (iii) and with it, the proof of the theorem.

REMARKS. In [1], Breuer and Gottlieb obtain an interesting extension of a comparison theorem of Levin [6] which they suggest bears on the Taam problem. This is not really the case, as they require for the application of their result that (1.2) have a positive solution $u$ with $u^{\prime}(t) \leqslant 0$. In the context of Case (i), this cannot occur.

\section{REFERENCES}

1. S. Breuer and D. Gottlieb, Hille-Wintner type oscillation criteria for linear ordinary differential equations of second order, Ann. Polon. Math. 30 (1975), 257-262.

2. G. J. Butler, On the oscillatory behavior of a second order nonlinear differential equation, Ann. Mat. Pura Appl. (4) 105 (1975), 73-92.

3. W. J. Coles, Oscillation criteria for nonlinear second order equations, Ann. Mat. Pura Appl. 82 (1969), 123-134.

4. P. Hartman, Ordinary differential equations, S. M. Hartman, Baltimore, 1973.

5. E. Hille, Non-oscillation theorems, Trans. Amer. Math. Soc. 64 (1948), 234-252.

6. A. Ju. Levin, A comparison principle for second order differential equations, Soviet Math. Dokl. 1 (1961), 1313-1316.

7. R. A. Moore, The behavior of solutions of a linear differential equation of second order, Pacific J. Math. 5 (1955), 125-145.

8. K. Schrader, Oscillation and comparison for second order differential equations, Proc. Amer. Math. Soc. 51 (1975), 131-136.

9. C. A. Swanson, Comparison and oscillation theory of linear differential equations, Academic Press, New York, 1968.

10. C. T. Taam, Non-oscillatory differential equations, Duke Math. J. 19 (1952), 493-497.

11. A. Wintner, On the comparison theorem of Kneser-Hille, Math. Scand. 5 (1957), 255-260.

12. J. S. W. Wong, On second order nonlinear oscillation, Funkcial. Ekvac. 11 (1968), 207-234.

Department of Mathematics, University of Alberta, Edmonton, Alberta, Canada T6G $2 \mathrm{Gl}$ 